\title{
Supercoil formation in DNA denaturation
}

\author{
A. Kabakçı̆ğlu \\ Department of Physics, Koç University, Sartyer, 34450 İstanbul, Turkey
}

E. Orlandini

Dipartimento di Fisica, CNISM and Sezione INFN, Universita' di Padova, Via Marzolo 8, 35131 Padova, Italy

D. Mukamel

Department of Physics of Complex Systems, The Weizmann Institute of Science, Rehovot 76100, Israel

(Received 19 November 2008; published 10 July 2009)

\begin{abstract}
We generalize the Poland-Scheraga model to the case of a circular DNA, taking into account the twisting of the two strains around each other. Guided by recent single-molecule experiments on DNA strands, we assume that the torsional stress induced by denaturation enforces the formation of supercoils whose writhe absorbs the linking number expelled by the loops. Our model predicts that when the entropy parameter of a loop satisfies $c \leq 2$, denaturation transition does not take place. On the other hand, for $c>2$, a first-order denaturation transition is consistent with our model and may take place in the actual system, as in the case with no supercoils. These results are in contrast with other treatments of circular DNA melting where denaturation is assumed to be accompanied by an increase in twist rather than writhe on the bound segments.
\end{abstract}

DOI: 10.1103/PhysRevE.80.010903

PACS number(s): 87.15.Zg, 36.20.Ey

Thermal denaturation of double stranded DNA [1] has been of recent interest due to its relevance to protein synthesis, polymerase chain reaction and microarray technologies. The denaturation transition has been extensively studied theoretically mainly by means of two models: $(a)$ the Poland-Scheraga (PS) model [2] which considers the opposite bases to be either bound with a certain energy gain or unbound (when part of a ssDNA loop) and ignores the twisting of the strands around each other, and $(b)$ the PeyrardBishop model [3] which is a one-dimensional model in which complementary bases interact by a distance-dependent potential. Both models have been used to study, e.g., the nature of the melting transition [2-5] and the bubble dynamics of the DNA [6-9]. It has recently been shown that the PS model predicts a first-order melting transition if one properly takes into account the self-avoidance of the chains $[4,10,11]$.

The original PS model treats the DNA as a long ladder, without considering the twisting of the two strains around each other. It can be argued that this feature is irrelevant for the thermodynamics of an open ended chain since the twisting strain can be released by the rotation of the chain ends. However, this assumption is no longer appropriate if the chain ends are not free to rotate or alternatively, for circular DNAs such as plasmids in bacteria. In this case, upon heating up to the melting temperature the two strands can no more fully depart from each other since the chemical bonds that assemble the sugar backbone are still intact. We assume topoisomerases and other topology modifying agents are not present in the solution.

Then, the partition function is restricted to a sum over configurations with a fixed linking number (the number of times one strand rotates around the other). A well-known theorem [12] states that the linking number (LN) is the sum of twist and writhe, where twist refers to the sum of the subsequent stacking angles along the DNA and writhe is associated with the geometry of the DNA's center line and measures the amount of twist absorbed by the excursions of the backbone.

Past attempts to include DNA's helicity in the PS model have considered a twisting strain associated with the modified stacking angle of subsequent base pairs accumulating upon loop formation. This assumption leads to the conclusion that the transition either changes its nature (becomes of higher order) or disappears altogether, depending on the treatment of the self-avoidance effects [13] and the applied external torsion [14]. In both works, as well as here, it is assumed that the LN accommodated by the loops is insignificant. The reason is that the single strands may be considered as random chains and their winding angle is rather small [15].

Recent experiments on single DNA chains, however, point to a different possible mechanism for the absorption of LN expelled from the denaturated loops. Experimental measurements of the torsional response of long strands under fixed stretching force $F_{s}$ show that when $F_{s}$ is small, the chain almost immediately undergoes a buckling transition, forming a supercoil that absorbs the externally introduced LN [16]. This mechanism of harboring the LN in the modified conformation of the center line (writhe) rather than in augmented base-pair stacking angles is a familiar phenomenon frequently observed in telephone chords. The presence of a similar phenomenon in DNA calls for a re-examination of thermal denaturation in a setting where the LN associated with the loops is transferred to supercoils formed by locally relaxed DNA segments. Indeed, recent experiments point to the presence of such a mechanism during plasmid denaturation $[17,18]$.

In Fig. 1 we depict the generalized PS model we consider for this purpose. In the model, a particular base pair may be either unbound (in a loop), bound in a coil, or bound in a supercoil. Unlike in the original PS model, a looped configuration is allowed only if the rest of the chain can be rearranged to form the required amount of supercoils for the 


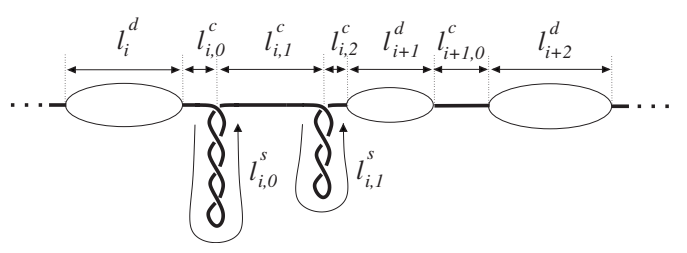

FIG. 1. A depiction of a microscopic configuration of the generalized Poland-Scheraga model discussed in the text. The superscripts $(d),(c)$, and $(s)$ refer to denaturation loop, coil, and supercoil regions, respectively.

conservation of the total LN. Let the linking number stored in the natural twist of a relaxed DNA in $\ell$ subsequent base pairs be accommodated in the writhe of a supercoil segment of total length $\ell^{\prime}=\delta \ell$. We will set $\delta=1$ below since a general treatment, although possible, does not bring new insight. Furthermore, it is reasonable to expect that loops form on the coils but not on the supercoils which are relatively rigid structures. As a result, formation or expansion of a loop on a coil is accompanied by an equal increase in the total length of supercoils in the system.

Let $E_{b}<0$ be the binding energy of bound pairs in the coil or supercoil state and $E_{s}>0$ be the cost of increasing the length of a supercoil by one bp (e.g., due to the bending rigidity). For simplicity, we assume the minimum size of a supercoil to be one base pair and one expects $E_{s}<-E_{b}$ for the DNA. The corresponding Boltzmann factors are $\omega$ $=\exp \left[-\beta E_{b}\right]$ and $\nu=\exp \left[-\beta\left(E_{b}+E_{s}\right)\right]$, where $\beta=1 / k T$. The Boltzmann factor corresponding to the configuration in Fig. 1 is then,

$$
\cdots \Omega\left(2 l_{i}^{d}\right) w^{l_{i, 0}^{c}} \nu^{l_{i, 0}^{s}} w^{l_{i, 1}^{c}} \nu^{l_{i, 1}^{s}} w^{l_{i, 2}^{c}} \Omega\left(2 l_{i+1}^{d}\right) w^{l_{i+1,0}^{c}} \Omega\left(2 l_{i+2}^{d}\right) \cdots .
$$

Here $l_{i}^{d}$ represents the length, in units of base pairs, of the loop $i$. The segment of bound pairs separating the $i$ and $i$ +1 loops is composed of alternating subsegments of coiled regions of length $l_{i j}^{c}$ and supercoiled regions of length $l_{i j}^{s}$. The entropic contribution of a loop of length $l$ is $\Omega(2 l) \equiv A s^{l} / l^{c}$, where $c$ is the universal entropic parameter of a loop, $s$ is a nonuniversal constant, and $A$ is a parameter which incorporates the cooperativity parameter, i.e., the Boltzmann weight associates with the initiation of a loop. Typically $A \simeq 10^{-4}$ and it is weakly temperature dependent [19]. Note that, some interloop regions may accommodate several supercoils, whereas some may have none.

Let $L_{D}, L_{C}$, and $L_{S}$ be the total length of the denaturated regions, the coiled, and the supercoiled regions, respectively, in a given configuration. The length of the molecule is $L$ $=L_{D}+L_{C}+L_{S}$. The canonical partition function is a sum over the contributions of all microscopic configurations with total DNA length $L$ and with a fixed LN, namely, with $L_{D}=L_{S}$. In the grand-canonical ensemble, the two constraints are relaxed by introducing two fugacities $\mu$ and $z$, which contribute an extra weight $z^{L} \mu^{L_{D}-L_{S}}$ to each configuration. The two fugacities are determined by taking the appropriate derivative of the grand partition sum $Q$,

$$
L=\frac{\partial \ln Q}{\partial \ln z}=\left\langle\sum_{i}\left[l_{i}^{d}+\sum_{j}\left(l_{i, j}^{c}+l_{i, j}^{s}\right)\right]\right\rangle,
$$

$$
0=\frac{\partial \ln Q}{\partial \ln \mu}=\left\langle\sum_{i}\left[l_{i}^{d}-\sum_{j} l_{i, j}^{s}\right]\right\rangle .
$$

Ignoring end effects which contribute terms of order $L$ to the partition sum, one finds that the grand partition function $Q$ can be expressed as

$$
\begin{aligned}
Q(z, \mu) & =\tilde{V}(z, \mu)+\tilde{V}(z, \mu) U(z \mu) \tilde{V}(z, \mu)+\tilde{V} U \tilde{V} U \tilde{V}+\cdots \\
& =\tilde{V} /(1-U \tilde{V}),
\end{aligned}
$$

where, following Ref. [4],

$$
\begin{gathered}
U(z \mu) \equiv \sum_{l=1}^{\infty} \Omega(2 l)(z \mu)^{l}=A \Phi_{c}(s z \mu) \\
\tilde{V}(z, \mu) \equiv V(z) /[1-W(z / \mu) V(z)],
\end{gathered}
$$

with

$$
\begin{gathered}
V(z) \equiv \sum_{l=1}^{\infty}(\omega z)^{l}=\omega z /(1-\omega z), \\
W(x) \equiv \sum_{l=1}^{\infty}(\nu x)^{l}=\frac{\nu x}{1-\nu x} .
\end{gathered}
$$

The functions $U, V$, and $W$ represent the grand sums for a loop, a coil, and a supercoil, respectively, and $\Phi_{c}(x)$ is the polylog function. Note that the functional form of the grand sum in Eq. (3) is similar to that of the original PolandScheraga model, except that the "propagator" for the coil regions is now dressed to accommodate an arbitrary number of supercoils. The price paid for conserving the linking number is that its associated fugacity $\mu$ needs to be calculated as a function of $z$ at each temperature.

After some algebra, Eq. (2) reduces to the more transparent relation $\frac{\partial W(z \mid \mu)}{\partial \mu}+\frac{\partial U(z \mu)}{\partial \mu}=0$. Using Eqs. (4) and (6), we obtain the following transcendental equation for $\mu(z)$ :

$$
\frac{\nu z}{(\mu-\nu z)^{2}}=\frac{A}{\mu} \Phi_{c-1}(s z \mu) .
$$

In order to study the nature of the denaturation transition (when it exists), we consider the thermodynamic limit ( $L$ $\rightarrow \infty)$ by focusing on the relevant pole of $Q(z)$ at $U\left(z^{*} \mu^{*}\right)$ $=1 / \tilde{V}\left(z^{*} / \mu^{*}\right)$, where $\mu^{*}=\mu\left(z^{*}\right)$ through Eq. (7). Substituting $1 / \tilde{V}=1 / V-W$, we obtain

$$
\left(\frac{1}{\omega z^{*}}-1\right)-\frac{\nu z^{*}}{\mu\left(z^{*}\right)-\nu z^{*}}=A \Phi_{c}\left[s z^{*} \mu\left(z^{*}\right)\right] .
$$

The average bound pair density in coiled and supercoiled segments is given by

$$
\theta_{c}=-\frac{\partial \log z^{*}}{\partial \log \omega}, \quad \theta_{s}=-\frac{\partial \log z^{*}}{\partial \log \nu},
$$

respectively. Therefore, a phase transition is associated with a singularity in the temperature dependence of the solution $z^{*}$ of Eqs. (7) and (8). 


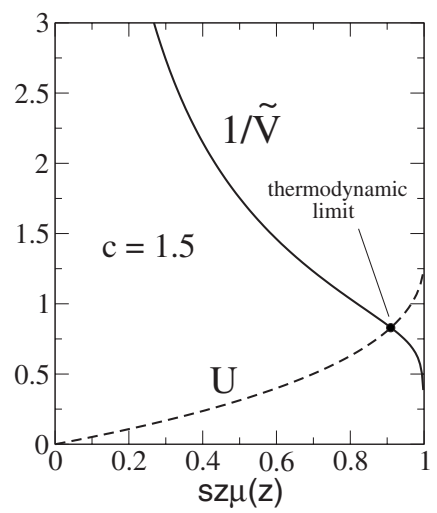

FIG. 2. Absence of a melting transition for $c<2$. The pole of the grand sum varies smoothly at all temperatures since $1 / V \rightarrow-\infty$ at $s z \mu=1$. The shown plot of $1 / \widetilde{V}$ is qualitatively the same for all temperatures.

Before proceeding, let us consider the simpler picture where the DNA chain is not circular but-nevertheless-can form supercoils. Assuming the ends are bound and free to rotate, we set $\mu=1$ in the grand sum since the system is now insensitive to the linking number. Equation (8) alone suffices to describe the phase transition in this case. Let the left-hand side and the right-hand side of Eq. (8) with $\mu=1$ be named $F(z)$ and $G(z)$, respectively. Then, $F(z)$ is a smooth monotonically decreasing function of $z$ for $0<z<1 / \nu$, with $F\left(0^{+}\right)=+\infty$. Similarly, $G(z)$ is a smooth monotonically increasing function of $z$ for $0<z \leq 1 / s$ (and divergent for $z$ $>1 / s)$, with $G(0)=0$. A phase transition exists if the smallest $z^{*}$ that satisfies Eq. (8) exhibits a singularity as a function of temperature. This is the case only if $c>1$ (so that $\Phi_{c}(s z)$ remains finite as $s z \rightarrow 1)$. In addition, one requires $F(1 / s)$ $>G(1 / s)$ at infinite temperature where $\omega=\nu=1$. Equivalently, after substitution,

$$
s-1 /(s-1)>1+A \zeta_{c},
$$

where $\zeta_{c} \equiv \Phi_{c}(1)$. Given a suitable set of the phenomenological constants that satisfy these conditions, the model exhibits a phase transition of first (second) order for $c>2 \quad(1<c$ $\leq 2$ ). This mechanism is described in detail in $[2,4]$.

Having established the existence of a phase transition in the unrestricted case and with the possibility of supercoils, let us now turn to the circular DNA, where $\mu$ is determined by Eq. (7) in order to ensure LN conservation. For a circular DNA, two regimes emerge.

For $\mathbf{1}<\mathbf{c} \leq \mathbf{2}$, a solution of Eqs. (7) and (8) with $s z \mu=1$ does not exist since Eq. (7) with $\Phi_{c-1}(1)=\infty$ dictates $\mu(z)$ $=\nu z$, whereas in Eq. (8), $\Phi_{c}(1)<\infty$. In fact, $1 / \tilde{V}(z, \mu) \rightarrow-\infty$ at $s z \mu=1$ for all temperatures, ensuring a smooth variation in $z^{*}$ as a function of temperature (Fig. 2). Hence, the secondorder melting transition found for the unrestricted DNA is absent when the linking number is conserved.

For $\mathbf{c}>\mathbf{2}$, substituting $s z^{*} \mu^{*}=1$ in Eqs. (7) and (8) and picking the smallest positive solution for $z^{*}$, one obtains

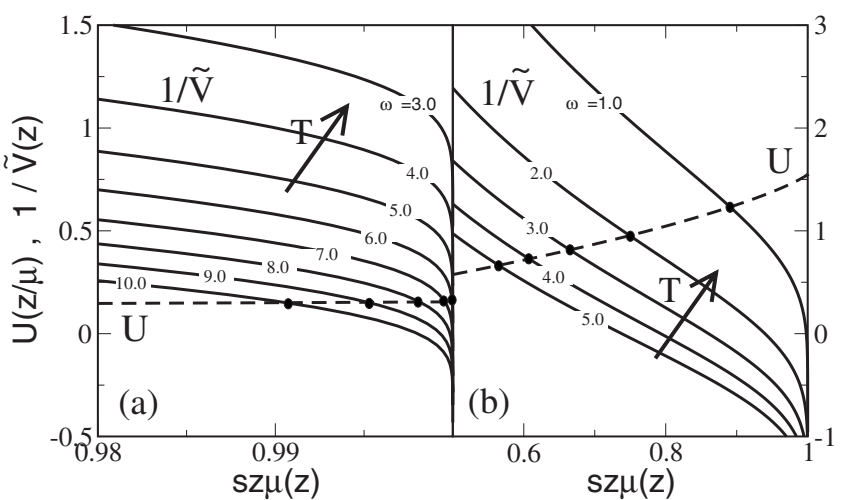

FIG. 3. Two melting scenarios in the model with $c=2.115, s$ =4.68, $E_{s}=0$, and (a) $A=0.1$ with a first-order transition at $\omega_{\text {crit }}$ $\simeq 4.18$ as found from Eq. (12); (b) $A=1.0$ with no phase transition. Full circles are the simultaneous solutions of Eqs. (7) and (8) corresponding to the thermodynamic limit. These parameter values which are relevant for self-avoiding walks on a cubic lattice [11] suitably demonstrate the two possible scenarios but are not intended to fit the experiments directly.

$$
z^{*}=\sqrt{\frac{1}{s \nu}} C_{-}, \quad \mu^{*}=\sqrt{\frac{\nu}{s}} C_{+},
$$

with $C_{ \pm} \equiv \sqrt{1+1 /\left(4 A \zeta_{c-1}\right)} \pm \sqrt{1 /\left(4 A \zeta_{c-1}\right)}$. The critical temperature is given by

$$
\frac{\nu_{c r i t}^{1 / 2}}{\omega_{c r i t}}=\frac{C_{-}}{\sqrt{s}}\left[1+A \zeta_{c}+C_{-} \sqrt{A \zeta_{c-1}}\right] .
$$

Since $\nu^{1 / 2} / \omega \geq 1$, a phase transition exists only if $s$ is sufficiently large. In particular, if

$$
s>A \zeta_{c-1},
$$

when $A \ll 1$. The transition temperature is reduced relative to the unrestricted case roughly by a factor $\sim \log _{s}\left(s / A \zeta_{c-1}\right)$. With $s \approx e^{12.5}$ as used by the MELTSIM (a widely used DNA melting simulator) scheme [20], one expects a transition to take place at a finite temperature (although the values of $s$ and $A$ optimized for our model may be different). The two qualitatively different regimes separated by the boundary in Eq. (13) are depicted in Fig. 3.

We conclude that the circular DNA undergoes a denaturation transition only if $c>2$. Performing a variational analysis near $s z^{*} \mu^{*}=1$, it is straightforward to show that the phase transition is first order for all values of $c>2$. Also note that the fraction of bound pairs in coiled and supercoiled segments $\theta_{c}^{>}$and $\theta_{s}^{>}$, in the high-temperature phase follow from Eqs. (9) and (11) as

$$
\theta_{c}^{>}=0, \quad \theta_{s}^{>}=1 / 2 .
$$

The high-temperature phase, although devoid of coiled regions, is not fully unbound. Half of the chain is in a supercoiled state while the remaining half unbinds, maximizing the amount of entropically favored loops.

These results contrast with the solution of the PS model without twist [4], as well as with the earlier calculations on the effect of locked-in twist on DNA melting transition 
$[13,14]$. The no-twist PS model predicts a second-order denaturation transition for $1<c \leq 2$; whereas if supercoil formation is taken into account, this second-order transition is absent when the $\mathrm{LN}$ is conserved. Earlier attempts to incorporate twist, where the loop formation is penalized by the overtwisting of the bound segments, found that the first-order transition which exists for $c>2$ in the original PS model becomes higher order or vanishes. In the proposed supercoiling scenario, the transition for $c>2$ remains first order.

Our findings should also apply to long DNA chains, where the twist expulsion through the ends may be hindered by kinetic effects (see, e.g., [21]). A more general framework where both supercoiling and twisting effects are incorporated is called for, in order to confirm the free energetic preference for the proposed mechanism over the alternative overtwisting scenarios.

We acknowledge helpful discussions with M. Baiesi, T. Garel, M. Hinczewski, Y. Kafri, A. Mostafazadeh, F. Ozturk, A. Stella, and C. Vanderzande. This work was partially supported by the Scientific and Technological Research Council of Turkey (TUBITAK) through the Grant No. TBAG$108 T 553$ and by the Minerva Foundation with funding from the German Ministry for Education and Research. We are indebted with ITAP for a school where the present collaboration with D.M. was initiated.
[1] R. M. Wartell and A. S. Benight, Phys. Rep. 126, 67 (1985).

[2] D. Poland and H. A. Scheraga, J. Chem. Phys 45, 1456 (1966); 45, 1464 (1966).

[3] M. Peyrard and A. R. Bishop, Phys. Rev. Lett. 62, 2755 (1989).

[4] Y. Kafri, D. Mukamel, and L. Peliti, Phys. Rev. Lett. 85, 4988 (2000).

[5] M. E. Fisher, J. Chem. Phys. 45, 1469 (1966); Y. Kafri, D. Mukamel, and L. Peliti, Eur. Phys. J. B 27, 135 (2002); M. Barbi, S. Lepri, M. Peyrard, and N. Theodorakopoulos, Phys. Rev. E 68, 061909 (2003).

[6] M. Barbi, S. Cocco, M. Peyrard, and S. Ruffo, J. Biol. Phys. 24, 97 (1999).

[7] A. Bar, Y. Kafri, and D. Mukamel, Phys. Rev. Lett. 98, 038103 (2007).

[8] H. Kunz, R. Livi, and A. Sütö, J. Stat. Mech.: Theory Exp. (2007) P06004.

[9] H. C. Fogedby and R. Metzler, Phys. Rev. Lett. 98, 070601 (2007).

[10] T. Garel, C. Monthus, and H. Orland, Europhys. Lett. 55, 132 (2001).
[11] E. Carlon, E. Orlandini, and A. L. Stella, Phys. Rev. Lett. 88, 198101 (2002); M. S. Causo, B. Coluzzi, and P. Grassberger, Phys. Rev. E 62, 3958 (2000).

[12] J. H. White, Am. J. Math. 91, 693 (1969); F. B. Fuller, Proc. Natl. Acad. Sci. U.S.A. 68, 815 (1971); G. Călugăreanu, Rev. Math. Pures Appl. 4, 5 (1959); G. Călugăreanu, Czech. Math. J. 11, 588 (1961).

[13] J. Rudnick and R. Bruinsma, Phys. Rev. E 65, 030902(R) (2002).

[14] T. Garel, H. Orland, and E. Yeramian, e-print arXiv:q-bio.BM/ 0407036.

[15] B. Drossel and M. Kardar, Phys. Rev. E 53, 5861 (1996).

[16] T. Strick, J.-F. Allemand, V. Croquette, and D. Bensimon, Prog. Biophys. Mol. Biol. 74, 115 (2000).

[17] L. Yan and H. Iwasaki, Jpn. J. Appl. Phys. 41, 7556 (2002).

[18] V. Víglaský, M. Antalík, J. Adamcík, and D. Podhradský, Nucleic Acids Res. 28, E51 (2000).

[19] R. Blossey and E. Carlon, Phys. Rev. E 68, 061911 (2003).

[20] T. Garel and H. Orland, Biopolymers 75, 453 (2004); R. D. Blake et al., Bioinformatics 15, 370 (1999).

[21] M. Baiesi and R. Livi, J. Phys. A 42, 082003 (2009). 